\title{
Air Transport Security and Crisis Management
}

\author{
Rudolf Volner \& Vladimír Smrž, \\ Technical University of Ostrava
}

\begin{abstract}
Network management is one of the key technologies needed to fulfill the requirements. In other words, a new paradigm for network management is also needed, in order to address the requirements arising both from the ATN side and the telecommunication side of the new telecommunication infrastructure. In particular, the new network management paradigm will incorporate the ATN management framework consisting of related standards, along with the telecommunication management framework, notably Telecommunication Management Network (TMN) standards from ITU-T.

\section{INTRODUCTION}

Civil aviation is characterized by constraints of different nature-impacting airlines, airport operators, and other actors intervening on the platform. We briefly isolated and illustrated these constraints by organizing them into two categories: regulatory constraints and operating constraints. The regulatory constraints refer to security and passengers economical rights. Security imposes certain obligations such as the respect of the security area integrity, reliability of the boarding pass, separation between screened passengers, and non-screened passengers and reconciliation, before take-off, between the passenger and his property. In addition, passengers have some economical rights that are concerning fulfillment of their contract of carriage.

Air traffic management is a broadly defined function which includes airspace management (ASM), Air Traffic Control (ATC), and Air Traffic Flow Management (ATFM). Its objective is to enable aircraft operators to meet their planned schedules of arrival and departure and adhere to preferred flight profiles.
\end{abstract}

Author's Current Address:

R. Volner, Ph.D., Departement of Civil Air Transport, Institute of Transport, Faculty of Mechanical Engineering VŠB -Technical university of Ostrava; and V. Smrž, Ph.D. Departement of Civil Air Transport, Institute of Transport, Faculty of Mechanical Engineering VŠB -Technical university of Ostrava.

Based on a presentation at the 2008 Carnahan Conference.

0885/8985/09/ \$25.00 USA @ 2009 IEEE
Air Traffic Flow Management (ATFM) is an important service, it aims to ensure an optimum flow of air traffic to or through airspaces during times when demand exceeds, or is expected to exceed, available capacity of the Air Traffic Control (ATC) system. The term ATFM is used to embrace any activity concerned with the organization and handling of the flow of air traffic in such a way that, while ensuring the safe, orderly, and expeditious flight of individual aircraft, the totality of the traffic handled at any given point, or in any area, is compatible with the capacity of the ATC system. The term ATC capacity reflects the ability of the ATC system or any of its subsystems or operating positions to provide service to aircraft during normal activities, and is expressed in numbers of aircraft entering a specified portion of the airspace in a given period of time. The maximum peak capacity which may be achieved for short periods may be appreciably higher than the sustainable capacity. ATFM supports ATC in meeting its main objectives of preventing collisions between aircraft, expediting and maintaining an orderly flow of air traffic, as well as of achieving the most efficient utilization of available airport and airspace capacity. Before starting the development process of the public air transport control and diagnostic system, passengers and maintenance personnel needs were analysed. Some of these needs were:

- modularity and flexibility of different software and hardware solutions,

- reducing causes of vandalism acts,

- introduce "black box" functionality into the control system,

- integrated passenger on-board and depot information system,

- system compatibility with other public transport, and

- rapid timetable deviation and expert model-based fault analyses. 


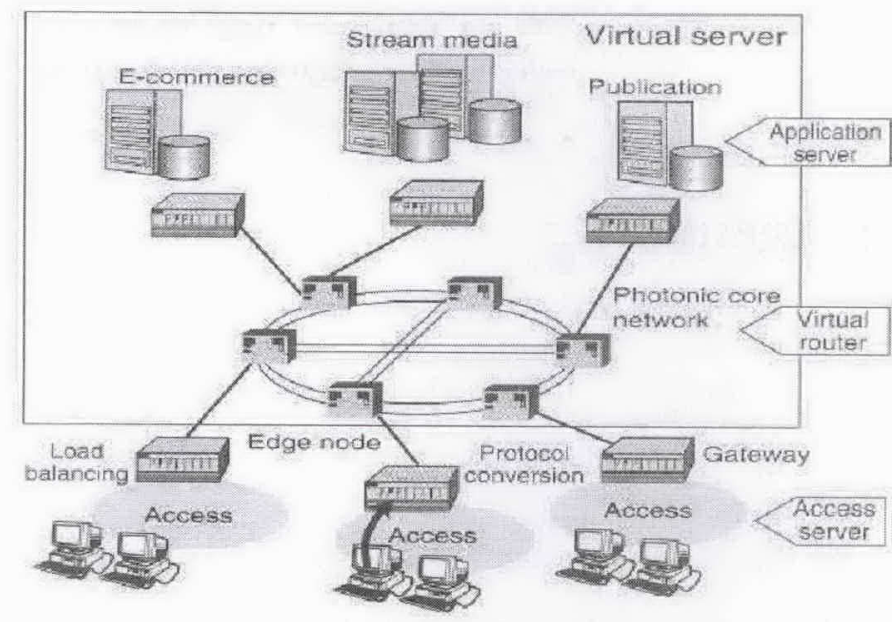

Fig. 1. The virtual server ATN management support

\section{NETWORK MANAGEMENT FOR THE NEW NETWORK SERVICE PARADIGM}

Encounters between the two paradigms have been one of the driving forces behind several important technical developments in late 1990s, voice-over IP, and Next Generation Network (NGN), to name a few. The same is true for network management, whose aim is to achieve high reliability and accountability of network operations and services through the use of Network Management System (NMS). In the early stages of the ATN, there was fairly clear separation between the ATN and telecommunication, both in terms of technologies and management responsibilities. The majority of traffic was still voice and the telecommunication network provided a WAN infrastructure, upon which the ATN was built as an overlay.

With the growth of the ATN, the volume of data traffic surpassed that of voice traffic and it has become common to build an all ATN-based backbone network, which is overlaid directly on SONET or Wavelength Division Multiplexing (WDM). These two networking paradigms - ATN and the telecommunication network - will merge into a new telecommunication infrastructure.

The future telecommunication service environment will look like a virtual network server, which consists of a set of application-specific servers running on ATN-based application protocols interconnected by a high-speed all optical network. The virtual server provides access to actual servers and service/network management support; see Figure 1.

In this new paradigm, network/service management itself is a service, provided by a set of specialized servers. Though it resembles the ATN, this new telecommunication must provide good characteristics as found in today's telecommunication network, i.e., high reliability and universal availability.

Network management is one of the key technologies needed to fulfill the requirements. In other words, a new paradigm for network management is also needed, in order to address the requirements arising both from the ATN side and the telecommunication side of the new telecommunication

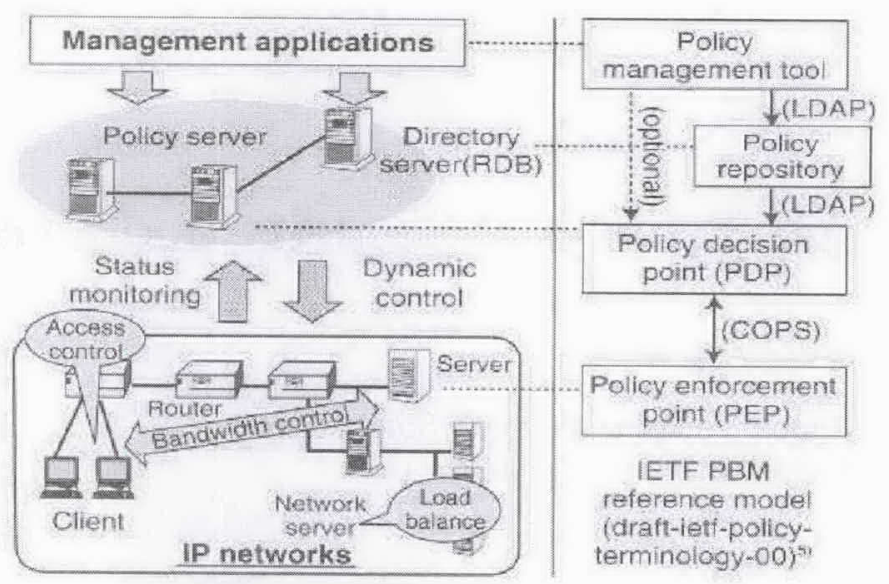

Fig. 2. ATN Engineering Task Force (ATNETF)

infrastructure. In particular, the new network management paradigm will incorporate the ATN management framework consisting of related standards, along with the telecommunication management framework, notably Telecommunication Management Network (TMN) standards from ITU-T.

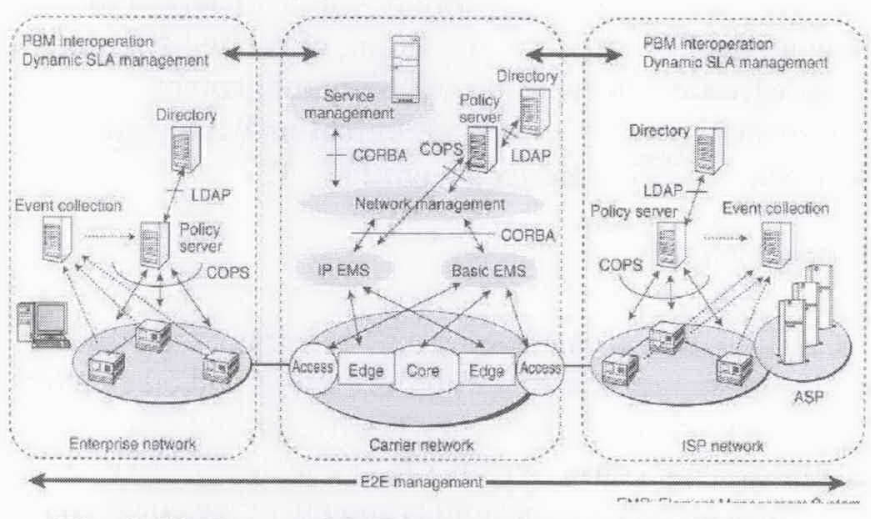

Fig. 3. End-to-end ATN-management architecture

\section{Policy-Based Management}

Policy-based management has attracted significant attention both from industry and the academic research community in recent years, as it has been recognized that PBM can effectively provide a good means to solve the puzzle of integrated ATN/telecom management; see Figure 2.

\section{End-to-End ATN-Policy Management Architecture}

In the emerging network service paradigm, end-to-end support both for network management and service management will be very important. The end-to-end ATN management should be able to provide guaranteed service, Quality of Service (QoS), Quality of Experience (QoE) for various ATN-based services, across administrative domains involved with the service provisioning. The new management paradigm should bring about smoother, more flexible, and more effective integration of service offerings from players in the new network service paradigm, including network providers, service providers Application Service Provider (ASPs) and end users; see Figure 3. 
The active policy augments the network intelligence of network Operation Support System (OSS) by adding more intelligent behavior at the service management layer.

\section{Safety Enhancements}

Providing internet access to airplanes when they are airborne opens up many possible service opportunities. Some are related to flight security and some are commercial in nature. Below is a list of possible service features that can be deployed in an internet-enabled airplane:

- using the internet access gateway as a beacon device -

when an airplane is airborne, it is very important for the administration to keep track of the position of the airplane. Typically, air traffic control keeps track of the flight position using transponders that detect a radio signal from the air route traffic control center or terminal radar control centers and respond with an amplified signal specifying crucial flight data including flight speed and height information. Although this system works efficiently, it is possible to turn off the transponder with the existing implementation. In fact, the pilot activates the transponder once the flight is airborne. This poses serious security threats as a hijacker, knowing the mechanism, can turn off the transponder and break communication between the ground station and the airborne flight,

- download of flight critical data in real-time the black box plays an important role in retrieving important data from a crashed airplane. A black box constitutes two parts:

- cockpit voice recorder (CVR), and

- flight data recorder (FDR).

As the name suggests, the CVR unit records the voice activity inside the cockpit and the FDR records the trivial data (like flight speed, altitude, temperature, etc.) related to the flight. Most of the black boxes used in today's airplanes are either made of magnetic tapes or solid-state memory boards. The magnetic tape based black boxes are being phased out and most of the solid-state memory board-based black boxes. With the existing computer hardware technology, it is possible to use a high capacity storage device within the airplane. This storage device can be connected to a server and also to the traditional black box/sensor network. One of the main advantages of using a server with the storage device attached is that the server can mirror all flight data to the ground station in real-time. This helps the ground station monitor the flight status/health and assist the flight crew in disaster situations,
- real-time video surveillance -

flight safety is one of the most widely discussed topics in the aviation industry. With the existing video compression standards, it is possible to transmit the video signals to the ground station in real-time. This enables the ground station to monitor the in-flight activities during abnormal situations and make appropriate decisions. This also helps the ground crew be prepared in the case of medical emergencies,

- remote controlling -

in remote situations where the flight crew is compromised or is unable to control the flight due to some unforeseen reasons, the ground station could enable remote controlling of the flight so that the flight could be landed safety on the ground. This feature will be helpful in the event of hijacks or medical emergencies.

\section{AVIATION SECURITY REQUIREMENTS}

Along with safety, the avionics subsystem must also possess sufficient security provisioning for a successful deployment. Adoption of open standards for data networks has further increased the security concerns. In addition, care must be taken about security requirements while achieving interoperability between various systems within the airplane.

In any network system, there are three basic security requirements that need to be addressed. They are confidentiality, authentication, and integrity. Data confidentiality ensures the privacy of end-users and protects their data from spoofing. Similarly, data integrity ensures that the data sent by the end-user is not modified by any malicious element in the network. Authentication is one of the most important factors in network security as it controls access to the network resources. Authentication ensures that only valid users have access to the network resources.

In addition to the above requirements, an airplane network needs additional security in terms of separation between various network segments. The control network has to be protected from unauthorized access. This requires the control network to be separated from the passenger network. Also, the passenger network resource usage needs to be monitored and controlled, which requires the passenger network to be connected to a gateway that performs both monitoring and controlling functions, in addition to providing Internet access.

\section{Security Issues}

The security issues involved with airplane networks can be broadly classified into two categories:

- external, and

- internal. 
The external security issues are mostly related to the external link connecting the airplane network with the ground station and to the protocol used to provide mobility support. The airplane network could be connected to the ground station by either satellite links or wireless links, depending on the location of the airplane. While the airplane is airborne, it could use either a satellite link or terrestrial link to connect to the ground station. If the airplane is within the range of a wireless access point, it could even use wireless media to connect to the external world. Each of these media has security issues associated with it.

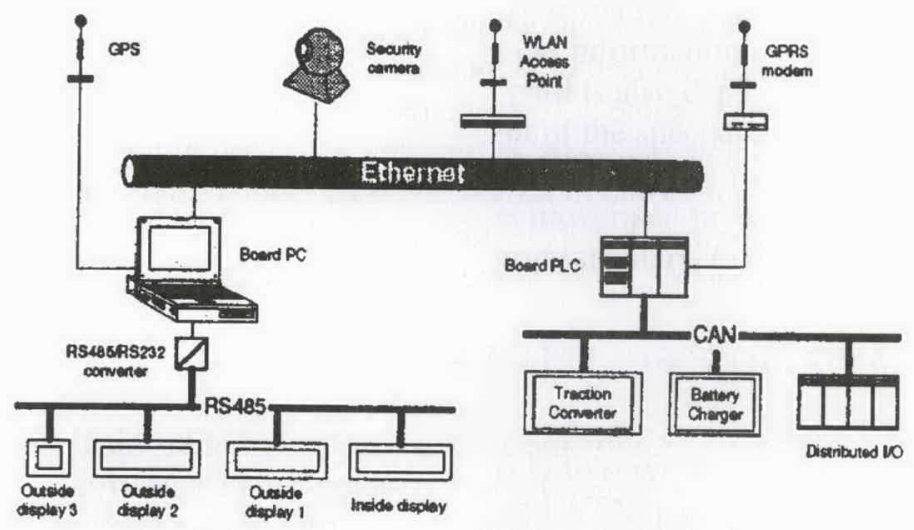

Fig. 4. Public air transport on-board control and diagnostics system

\section{AVIONICS SUBSYSTEM DESIGN}

The main requirements of the avionics subsystems are high determinism and low response time. Different layer 2 technologies like Ethernet, ATM, and fibre channel could be considered to provide such a high determinism and low response time. Ethernet is one of the strongest contenders for connections between various flight subsystems and is also mentioned in standards.

\section{Avionics Data Network Design}

Security and quality of service are the two important parameters that need to be considered while designing the aviation data networks. The quality of service here does not reflect the quality of service requirements of the end-user applications, but it represents the requirements of the avionics subsystem itself.

One of the major security requirements of aviation data networks is the separation of different network subsystems. An aviation data network could possibly contain three major network segments; namely, a control network, crew network, and passenger network. As the names suggest, the control network predominantly consists of avionics components.

The crew network is used by the flight crew for monitoring purposes and the passenger network enables internet connectivity for the passengers.
In order to protect the control network from unauthorized access and security attacks, it is necessary that the control network is separated from rest of the aviation data networks.

The separation can either be logical or physical. The network activity monitoring/controlling server could be built in line with an Intrusion Detection System (IDS).

However, unlike normal IDS, the network activity monitoring/controlling server can make decisions based on the data feed from many sources including the cabin voice recorder and surveillance equipment placed at strategic locations within the flight. The server could potentially control all of the active forwarding devices. Depending upon the network activity and the security status of the aviation data network, the server can reconfigure the active devices and facilitate control network traffic during emergency situations.

\section{SYSTEM ATN}

The control and diagnostic system is divided into the on-board; see Figure 4. Messages that are transmitted between an airplane and the dispatching centre have different priority levels. High priority messages should be transmitted immediately, while others can be transmitted periodically or when a connection is possible.

\section{The Diagnostic System has a Hierarchical Structure}

Different diagnostic methods and functions are integrated into the system, depending on the priority and system hierarchy level. Functionality of the diagnostic system is distributed between several devices. The traffic supervision and maintenance expert system is used in the diagnostic, control, dispatching, economic, and maintenance process. For example, some functions of the on-board diagnostic system are:

- built-in self/tests of the complete airplane,

- event recording, and

- fault detection, presentation, corrective actions, interlocking.

The functions of the maintenance expert system in the dispatching center are:

- support for preventive, corrective, and conditional maintenance,

- analysis of event recorder data and fault database,

- remote diagnostic/fault elimination via wireless data link,

- knowledge-based systems for trouble-shooting, etc. 


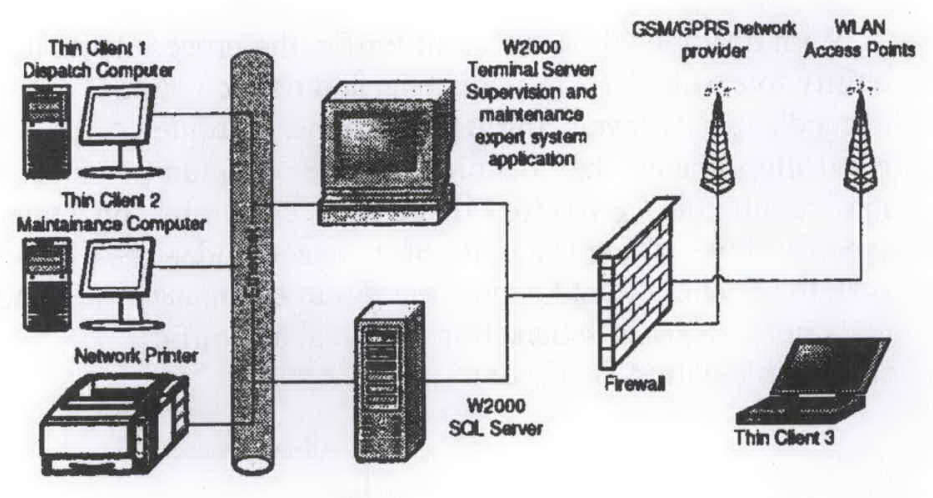

Fig. 5. Structure of dispatching center

\section{Behavioural Analysis of the Tower Controller Activity}

The observation was based on the camera recordings and the audio of the controller; there were no recordings of the pilots' communication available. The camera was placed slightly aside of the controller, out of his line of sight.

The recording involves two controllers working at the tower control position. The first controller was filmed from the beginning of his shift up to the hand off (50 minutes), whereas the following controller was filmed only at the beginning of his shift (remained 10 minutes).

The amount of collected data for both controllers was not equal; therefore we did not carry out a comparison between the performances of both controllers.

The observation exclusively concerned the behavioural activity of the controller. The following activities were distinguished to describe the controller's performance:

\section{- Window:}

when the controller was looking outside the window in front of his position. As a subcategory there is the usage of binoculars marked, describing a period when the controller is supporting vision with binoculars.

\section{- Radar:}

describes the time when the controller was looking or manipulating with a radar or screen providing meteorological information.

\section{- Strips:}

describe the time when the controller was scanning, ordering, or writing on the strips.

\section{- Strips delivery:}

describe the time when the controller was out of the working volume, allowing him to scan the window in order to provide strips to other positions.

\section{- Coordination:}

describes the activity when the controller discussed and arranged traffic between the Ground control, Supervisors, or Flight Data Assistant.

\section{- Clearance:}

describes issuing clearances. There were two kinds of instructions remarked:

- Landing describes issuing instructions for aircraft that are still airborne ("clear to land" and "continue approach").

- Runway vacated describes issuing instruction to aircraft that already landed ("contact ground").

\section{- Non-active:}

describes the time when a controller was not occupied with control activity. Due to low level of traffic, he was involved in other activities (chatting with others, resting) while remaining in his position.

- We used the Interact software to record parameters of activities, produce graphic representation of data, and obtain basic statistic analysis.

For the analysis, the frequency of occurrences and duration of activities were measured. Frequency is calculated as a total number of occurrences and duration is the time of the activity. One occurrence of a particular activity is defined as continuous action without interruption. Any distraction was treated as the suspension of action and started a new occurrence. The information provided by radar including the screen with meteorological conditions for manipulating with strips required head-down time, whereas looking outside is head-up activity.

The controller regularly refocuses his vision between direct observation at great distance and the radar image on his desk.

The activity placed in a column will be followed by an activity placed in a row with the given probability. The highest probabilities are highlighted in red. The transition probability indicates that the controller was constantly switching his attention between the radar, window, and strips.

Additionally, we did not define any special pattern for airborne and landed aircraft as seen from the two last rows of the table.

\section{Tracking Requirements for}

\section{ATC Tower AR Environments}

The typical taxonomy for tracking is based on technology. There are seven principal categories of tracking technologies.

An appropriate tracking system must meet the requirements of an air traffic control tower. First, the sensitive surveillance and communication equipment in the tower does not allow sources of potentially disturbing 
exogenous signals. Second, the tower personnel must be free to perform their activities unhindered by the tracking system. For this reason the tracking system must have a natural source and be non-intrusive.

A tracking system relies on a source emitting a signal perceived by a sensor. An artificial source may be emitting a magnetic field, rays of light, or sound waves. A natural source, on the other hand, may be Earth's field of gravity or a subject's muscles. A natural source tends to be permanent and omnipresent and is therefore accepted in the tower.

An implementation of mechanical tracking would be a so-called "boom," which is a cantilever-mounted display tracking the user's field of view (FOV). Due to its large form factor, this must be considered as too intrusive.

Body-mounted exoskeletons cannot convey orientation unless all joints from the feet and up are implemented. In addition to being inarguably intrusive, the tracking system must be accompanied by extra sensors for orientation, a set-up which is rather to be considered as a hybrid system. If the intrusiveness criterion were relaxed to allow small sensors on the body, inertial tracking could have been considered, but inertial tracking exhibits drift, poor signal-to-noise ratio (SNR) at low motion frequencies, and can only provide relative positioning.

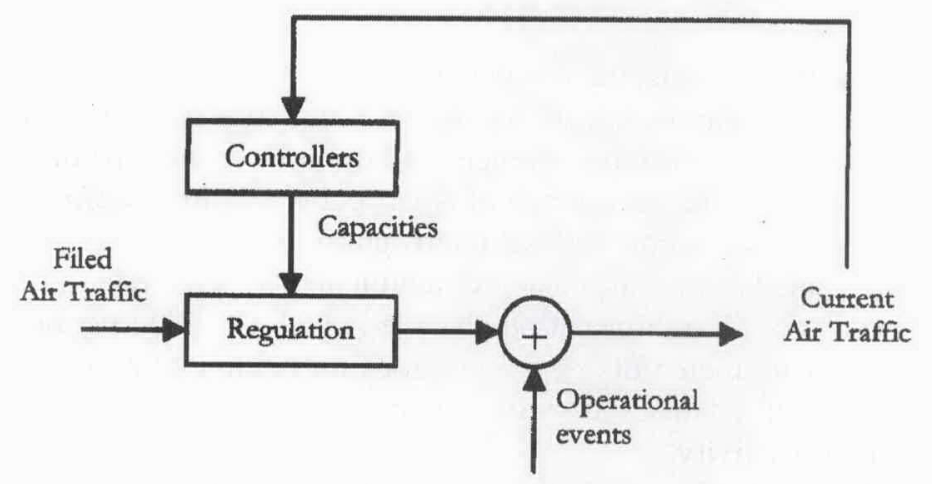

Fig. 6. Current implementation of ATFM

\section{Air Traffic Flow Management Pre-Tactical Planning}

Balancing in the European ATM system consists in ground delay programs, aircraft may be affected by regulation delay at departure airports in order to satisfy capacity constraints of arrival airport and airspace sectors throughout the trajectory. The Central Flow Management Unit, located in Brussels, uses the flight plans already submitted to measure magnitudes of traffic volumes in the system then generates the required amounts of delay.

Flight plans are submitted at least three hours before the estimated off-block time, when regulation starts at about two hours before off-block time, the required delay is continuously (i.e., periodically) updated according to the global air traffic demands and the capacity limitations activated by ATC.

Priority is given to the first aircraft planned to enter the sector, as equity suggests.
With operational events of air traffic, the process loses its ability to ensure ATC requirements and traffic volumes can exceed capacity levels. In such situations, en-route controllers change their opening scheme configuration and may require the CFMU to activate some constraints on some specific flows. There is a trade-off between under-use of system resources (i.e., keeping margin in occupancy rate) and real-time constraint satisfaction (i.e., real air traffic compatible with ATC needs).

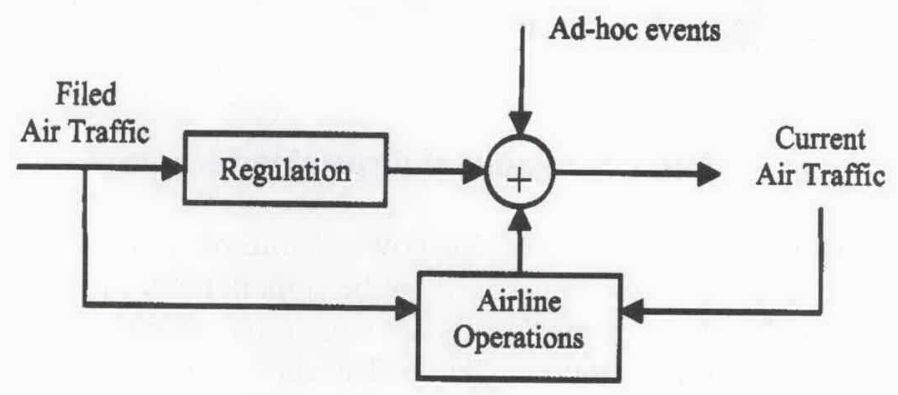

Fig. 7. Airline operation effect on ATFM

Previous description is depicted in Figure 6, now there are two important remarks:

- Perturbations are not independent from the input signal, and

- Regulation is made on the basis of flight plans already submitted, under assumption that flights are all on time (or have negligible delays).

The primary purpose of aircraft operations control is to ensure the optimum operation of flights planned for a certain defined period ahead of departure and until their completion, and to coordinate with all concerned, and, finally, to disseminate information about any measures taken in case of irregularities. Within an airline organization, this centralized function is carried out by the Airline Operations Center (AOC) and its main objectives are: to keep the flight program as close as possible to the published schedule; and minimize the effects of any irregularities in accordance with company policy. In so doing, the AOC will keep the various operational departments concerned informed about the movement of aircraft, e.g., flight operations, engineering control, passenger, and airport.

Accurate and timely information concerning aircraft departures and arrivals, and variations to the planned flight programs, such as delays, diversion, cancellations, reroutings, and change of aircraft type, will enable the operational departments to coordinate their activities in the best interests of airline customers, thereby enhancing flight safety, punctuality and regularity, and reducing costs.

To control effectively a large fleet of aircraft for a worldwide operation is a very complex undertaking. Today, computerized systems process and organize the immense 
amount of data involved and provide the Airline Operations Centers with a better knowledge of the total situation and the libely consequences involved. As a result, this improves the operational decision-making process which, in turn, can contribute significantly to efficiency and profitability of airline operations.

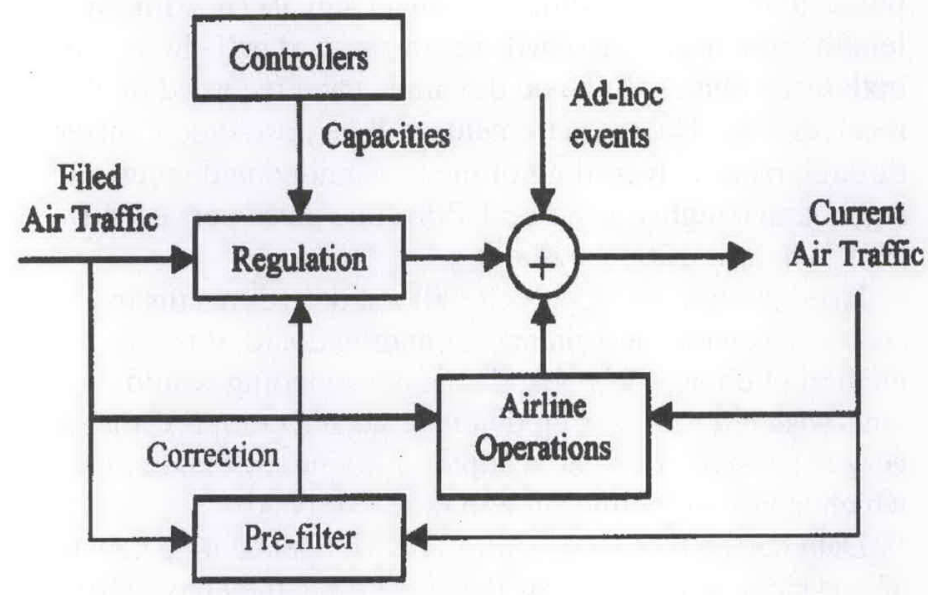

Fig. 8. Proposed design for ATFM

As depicted in Figure 7, dispatchers in airline operation centers use aircraft which may be delayed because of some ad-hoc event, in order to recover the nominal schedule of services or reduce the costs of irregularities. This dynamic change in demand, depending on the real state of air traffic is not considered in the current ATFM implementation, so it's a supplementary perturbation to regulation output. Notice that ad-hoc events in Figure 7 can be supposed to be independent of the system input.

Better regulation can be achieved by pre-filtering air traffic demand. The pre-filter has the duty to anticipate short-time change in flight times from the current state of air traffic, which allows getting reliable information about sector loads during the stage of regulation.

Uncertainty is inherent in Air Traffic Management, if it's impossible to foresee an aircraft failure or to prevent bad meteorological conditions, delays spoiling the series of flights because of propagation and airline operational control policies can be estimated by modelling the behavior of dispatchers. The role of the pre-filter is to compensate the disruptions resulting from OCC tactical actions.

Because of the complexity of this task, we restrict the scope to the phenomenon of delay propagation throughout the daily schedule of an aircraft, as follows.

By analogy with updating flight strips once aircraft are airborne, which have been adequately fulfilled, it's plausible to extend the range of update beyond next ground operations if we get the necessary pieces of information. It is not quite as accurate as current strip update, because taxi time is somewhat variable and the ground block span may contain a margin used as an absorber in regard to delay. But even so, confidence intervals can be provided as prediction inference.

Pre-filtering needs to have knowledge of the allocation between aircraft and flights, flights A and B of Figure 9 relate to the same plane. It's a pity that flight plans mention call signs with aircraft types but no information about aircraft tail numbers. Since that's how it is, airlines are able to substitute for aircraft of the same type in order to minimize the costs of delays without filing new flight plans, imperiously three hours before the estimated off-block times. A peaceful way to undertake this problem consists in trying to match the flights accomplished by the same aircraft on the basis of flight plans data.

For the purpose of clarity, we are going to provide a real example of pre-filtering based on coupling departure with arrival flights. However the general inference process remains beyond the scope of this paper, as mentioned before, because of the diversity of phenomena, airlines characteristics, and behaviors. This matter is still under investigation.

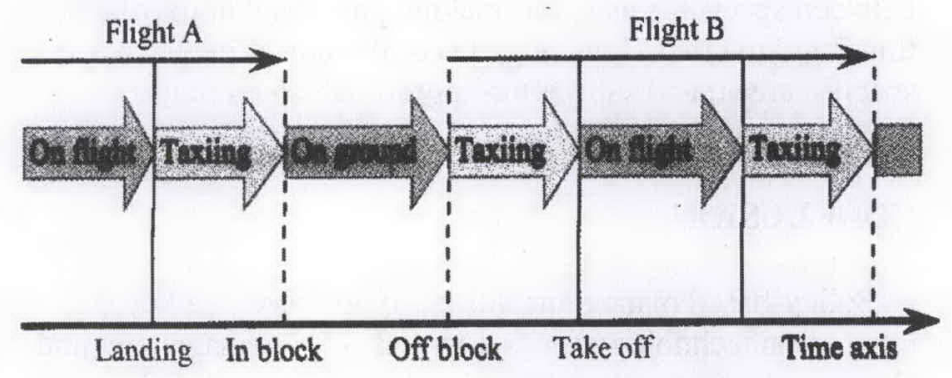

Fig. 9. Aircraft lifecycle

\section{CRISIS MANAGEMENT}

Security and crisis management issues are of paramount importance for the success of human events. Citizen's confidence level, as far as the ability of organizers to provide security is concerned, is a factor directly impacting their presence. Considering the success of air transport events depends, to a great extent, on the security level provided, automated systems for crisis prevention and management are expected to have wide application. Air transport-related crimes, however, require sophisticated plans. Managing crisis situations during large scale air transport events means to adopt an integrated system which incorporates intelligent access control and spectator tracking systems.

Modem biometric technologies provide enhanced security levels by introducing a new dimension in the authentication process called "proof by property." However, the design and deployment of a security architecture incorporating biometric technologies hides many pitfalls, which when underestimated can lead to major security weaknesses. Although biometrics have been deployed in pilot systems for protecting access to air transport events in the past, no integrated solution has been proposed taking into account the related security standards and no complete studies have ever proven the benefits of such deployments.

\section{Intelligent Access Control System}

The basic component of the system is the biometric access control system for the access control of spectators and 
accredited persons. This system minimizes security risks and offers the possibility to intelligently control the throughput rate, the stadium completeness, monitor the movement of spectators and accredited persons within the stadium premises, and remotely control the gates. The strength of the system is centered on the indubitable biometric identification method (fingerprint scanning), complemented by the ease of use offered by the RFID technology and the portability resulting from the use of already available mobile communications infrastructures (GSM, GPRS, or WiFi). The smart card is used not only to secure access to physical resources, but also to store data about the cardholder as well as a limited set of critical medical information (Emergency Data Set) or provide the key to access information stored in a remote database. The RFID smart card is also deployed for monitoring the statistical movement of the spectators between specific zones, for tracking patterns that may be translated to crisis signs (e.g., mass movement between specific areas) and submit the appropriate alerts to the Coordination Centre.

\section{CONCLUSION}

Policy-based management is likely to serve as a key integration technology for end-to-end ATN management and also for telecommunication network. Activity policy is a relatively new research area, which is to be explored in the coming years.

The new system gives the following advantages:

- many functions can be integrated into the multi-processor system for optimum cost-effectiveness,

- modular design allows flexible addition of components with minimum expense for extra hardware or software,

- redundancy can be incorporated simply and inexpensively, and

- high reliability.

Data network-enabled aircrafts have opened up a new set of service opportunities. At the same time, they have also introduced several security threats that need to be addressed. These security threats can originate from outside the airplane or from within the plane. Policy-based management is likely to serve as a key integration technology for end-to-end ATN management and also for telecommunication networks. Activity policy is a relatively new research area, which is to be explored in the coming years.

Biometrics security system enabled air transport have opened up a new set of service opportunities. At the same time, they have also introduced several security threats that need to be addressed. These security threats can originate from outside the airplane or from within the plane.

The chosen method of measurement is as follows: Narrowband measurements (within one $8.33 \mathrm{kHz}$ channel) from base band LF audio to base band LF audio will be pursued. Pre-computed measurement signals (maximum length sequence, chirp,) will be transmitted half-duplex in both directions (uplink and downlink) and recorded on the receiver side. The measurements will be post-synchronized through parallel recording of the transmitted and received signal and a highly accurate 1 PPS (one pulse per second) GPS synchronization signal.

Even though this approach will not deliver results as accurate as wideband channel sounding could, it is the method of choice. Wideband channel sounding would otherwise require a set-up that is large and complex, that is very expensive, and that occupies a frequency bandwidth which is virtually unfeasible in the VHF band.

Data network enabled aircrafts have opened up a new set of service opportunities. At the same time, they have also introduced several security threats that need to be outside the airplane or from within the plane.

\section{REFERENCES}

[1] Ragothaman, V., Thanthry, N., Bhagavathula, R. and Pendse, R.., IP Connectivity and DAP, In the Proceedings or $23^{\text {rd }}$ Digital Avionics System Conference, Salt Lake City, Utah, October 2004.

[2] Volner, R. and Boreš, P., ATN Networking - Policy based Management for Enterprise and Carrier,

Poster Abstract of the $27^{\text {th }}$ International Conference on Information Technology Interfaces ITI 2005,

Cavtat/Dubrovnik, Croatia, June 2005, pp. 37-38, ISBN 953-7138-04-6

[3] Volner, R.,

Aviation Data Networks - New Avenues for Flight Safety, Security Issues and Network Architecture, Poster Abstract of the $27^{h}$ International Conference on Information Technology Interfaces ITI 2005, Cavtat/Dubrovnik, Croatia, June 2005, pp. 35-36, ISBN 953- 7138-04-6

[4] Thanthry, N. and Pendse, R., Aviation Data Networks Security Issues and Network Architecture, In the Proceedings of $38^{\text {th }}$ International Carnahan Conference On Security Technology, Albuquerque, New Mexico, October 2004.

[5] Dimitriadis, C. and Polemi, D., Biometrics - Risks and. Controls, Information Systems Controll Journal (ISACA), Vol. 4., (2004), 41-43. 